\title{
Differenzanalysen: Gender-Forschung und interkulturelle Literaturwissenschaft in Deutschland
}

\section{Ortrud Gutjahr (Hamburg)}

In den letzten Dezennien des 20. Jahrhunderts hat innerhalb der Germanistik in Deutschland ein wissenschaftlicher Paradigmenwechsel mit weitreichenden Folgen stattgefunden. Die kulturwissenschaftliche Wende in den Geisteswissenschaften, die durch die vom Wissenschaftsrat befürworteten und von der Deutschen Forschungsgemeinschaft seit Beginn der 90er Jahre geförderten Reforminitiativen zur Umstrukturierung der Geistes- in Kulturwissenschaften markiert ist, hat zur Etablierung neuer Forschungsrichtungen geführt. Neue Studiengänge sind entstanden und wissenschaftliche Publikationen tragen dem aufgeflammten Interesse an Kulturtheorien und kulturwissenschaftlichen Fragestellungen in großem Ausmaß Rechnung. Innerhalb dieses Veränderungsprozesses wurden Gender und Interkulturalität zu Forschungsparadigmen, die in verschiedenen Wissenschaftsdisziplinen Karriere machten und zur Etablierung neuer Studienrichtungen führten. So wird heute ganz selbstverständlich beispielsweise von einer Gender-Forschung in der Theologie oder Kunstgeschichte wie auch einer interkulturellen Philosophie oder Pädagogik gesprochen. Auch für die Literaturwissenschaft erhielten beide Forschungsrichtungen eine zentrale Bedeutung.

Zur Gender-Forschung wurden Zentren gegründet, Professuren besetzt, Studiengänge konzipiert und Graduiertenkollegs eingerichtet. Mit dem Forschungsparadigma Gender wurden bereits bestehende Ansätze der Frauenforschung und feministischen Literaturwissenschaft einer kritischen Revision unterzogen und zahlreiche Publikationen haben die Frage nach der Konstruktion von Geschlecht mit neuen kulturwissenschaftlichen Ansätzen verknüpft. Zur gleichen Zeit wurde auch Interkulturalität innerhalb der Germanistik zu einem forschungsleitenden Begriff. Es wurde eine Gesellschaft für interkulturelle Germanistik gegründet, die mit ihren Tagungen und regelmäßig erscheinenden Publikationen ein breitgefächertes Spektrum an Fragestellungen, Themen und Methoden zur 
interkulturellen Literaturwissenschaft vorstellt. An verschiedenen deutschen Universitäten wurden Professuren für interkulturelle Germanistik oder interkulturelle Literaturwissenschaft eingerichtet, Schwerpunkte zur interkulturellen Literaturwissenschaft in germanistische Studiengänge integriert und Graduiertenkollegs zu interkulturellen Themen und Fragestellungen gefördert. Es ist eine Flut von wissenschaftlichen Publikationen erschienen, die das neue Forschungsgebiet innerhalb der Literaturwissenschaft elaborieren. Die Entwicklung in Deutschland innerhalb der Germanistik, Fragestellungen und Methoden zu Gender und Interkulturalität in Forschung und Lehre zu etablieren, ist Teil eines fächer- und disziplinenübergreifenden Trends, der zu neuen Untersuchungsansätzen geführt hat. Gender und Interkulturalität sind dabei Paradigmen, die sich aus unterschiedlichen Wissenschaftstraditionen heraus entwickelt haben, sich jedoch etwa zeitgleich etablieren konnten. Gender-Forschung und interkulturelle Literaturwissenschaft haben in ihrer Frage nach den Bedingungen von Differenzbildungen signifikante Berührungspunkte, die für eine Erweiterung und Weiterentwicklung beider Ansätze fruchtbar gemacht werden können. Ich möchte im Folgenden deshalb zunächst in einem historischen Überblick die Entwicklung und Herausbildung der beiden Wissenschaftsparadigmen hinsichtlich ihrer Ansätze zur Differenzkonstruktion nachzeichnen, um daran anschließend die Verbindung von Gender und Interkulturalität im Rahmen der kulturwissenschaftlichen Wende zu einem neuen Forschungsansatz umreißen zu können.

Die kulturwissenschaftliche Wende koinzidiert mit einer umfassenden Selbstreflexion der feministischen Forschung, wie sie sich seit den 70er Jahren des zwanzigsten Jahrhunderts an den deutschen Universitäten etabliert hatte. Die begrifflichen Grundlagen der feministischen Forschung wurden überdacht. und die langjährigen Debatten um Gleichheit

\footnotetext{
${ }^{1}$ Vgl. hierzu u. a.: Katharina Baisch, Ines Kappert, Marianne Schuller, Elisabeth Strowick, Ortrud Gutjahr (Hg.): Gender Revisited. Subjekt- und Politikbegriffe in Kultur und Medien. Stuttgart: Metzler, 2002. Ingrid Bauer (Hg.): Gender-Studies: Denkachsen und Perspektiven der Geschlechterforschung. Innsbruck: Studien Verlag 2002. Stella Jegher: Feminismus, Gender, Geschlecht, Zürich: Widerspruch 2003. Ingrid Neumann-Holzschuh (Hg.): Gender, Genre, Geschlecht: sprach- und literaturwissenschaftliche Beiträge zur Gender-Forschung. Tübingen: Stauffenburg 2001. Ewa Waniek, Silvia Stoller (Hg.): Verhandlungen des Geschlechts: zur Konstruktivismusdebatte in der Gender-Theorie. Wien: Turia + Kant 2001.
} 
oder Differenz wurden verschoben auf die Reflexion der Kategorie Geschlecht selbst. ${ }^{2}$ In diesem Zusammenhang setzte nun auch eine intensive Beschäftigung mit der in Amerika entwickelten Gender-Forschung ein, ${ }^{3}$ die nicht ohne Grund erst mit Verzögerung die deutsche Theoriebildung beeinflusste. Denn erst durch die kulturwissenschaftlichen Fragestellungen und Ansätze eröffneten sich auch für die Untersuchung der Geschlechterkonstruktionen neue Perspektiven. Die Sex/Gender-Relation, die eine Unterscheidung zwischen biologischem und sozialem Geschlecht ermöglicht, wurde für die Diskussion um die Kategorie Geschlecht zentral. Vor allem aber wurden nun Grundannahmen der Geschlechterforschung hinterfragt, wie sie auch von der feministischen Literaturwissenschaft in Deutschland über lange Jahre mitgetragen worden waren.

Die feministische Literaturwissenschaft, die im Zuge der Studentenbewegung und ihren vielfältigen Emanzipationsansprüchen innerhalb der Germanistik entstanden war, ging mit einem geschlechtsspezifischen Epochenumbruch im Bildungssektor einher. Erstmals in der Geschichte der deutschen Universität nahm in den 60er Jahren des 20. Jahrhunderts auch eine größere Anzahl von Studentinnen das Studium auf und organisierte sich politisch, um die Repräsentanz eigener Fragestellungen in Forschung und Lehre anzumahnen. Die feministische Literaturwissenschaft etablierte sich zunächst als "Wissenschaft am Rand," denn zumeist wurden Themen und Fragestellungen der feministischen Bewegung in studentischen Arbeitsgruppen oder auch außeruniversitären Erfahrungsgruppen ventiliert. So wurden Texte von Autorinnen im Kontext der ersten Frauenbewegung um 1900 gelesen, wie beispielsweise die von

\footnotetext{
2 Inge Stephan: "Gender, Geschlecht und Theorie." In: Christina von Braun und Dies. (Hg.): Genderstudien. Eine Einfuihrung. Stuttgart: Metzler, 2000, 58-96. Claudia Breger, Dorothea Dornhof, Dagmar von Hoff: "Gender Studies, Gender Trouble. Tendenzen und Perspektiven der deutschsprachigen Forschung." In: Zeitschrift für Germanistik NF 9.1 (1999), 72-113.

${ }^{3}$ Linda J. Nicholson: Gender and History: the Limits of Social Theory in the Age of the Family. New York: Columbia UP 1986. Nancy Fraser: Unruly practices: Power, Discourse, and Gender in Contemporary Social Theory. Oxford: Minneapolis: University of Minnesota Press 1989. Elaine Showalter: Speaking of Gender. New York: Routledge 1989. Mary Roth Walsh (Hg.): Women, Men, and Gender. Ongoing Debates. New Haven und London: Yale UP 1997.
} 
Verlage reagierten rasch auf diesen neuen Trend und richteten eigens Reihen für die so genannte "Frauenliteratur" ein. Die populärsten Beispiele dieser neuen Literatur waren Karin Strucks Klassenliebe (1973), Verena Stefans Häutungen (1975) und Brigitte Schwaigers Wie kommt das Salz ins Meer? (1977). ${ }^{11}$ Im Zuge dieser Rezeption der Frauenliteratur wurde gefragt, ob es für Autorinnen möglich sei, einen anderen Blick auf die Geschlechterordnung zu werfen, oder gar eine weibliche Ästhetik zu entwickeln. ${ }^{12}$ Während in der englischsprachigen Literatur Schriftstellerinnen kanonisiert sind, ${ }^{13}$ mussten mit der feministischen Literaturwissenschaft in Deutschland nicht-kanonisierte Autorinnen durch Relektüre erst wieder ins Bewusstsein gehoben werden. Insbesondere Werke von Autorinnen der Gegenwartsliteratur, die sich mit den Zumutungen und Verwerfungen weiblicher Rollenzuschreibungen auseinander setzten, wie Unica Zürn, ${ }^{14}$ Marleen Haushofer ${ }^{15}$ und vor allem Ingeborg Bachmann, ${ }^{16}$

"Vgl. hierzu meinen Beitrag: "Immigration in den imaginären Körper. Die Frauenliteratur der siebziger Jahre." In: Johannes Cremerius u. a. (Hg.): Freiburger literaturpsychologische Gespräche. Bd. 12: Literarische Entwürfe weiblicher Sexualität. Würzburg: Königshausen \& Neumann 1993, 279-303.

${ }^{12}$ Sigrid Weigel: Die Stimme der Medusa. Schreibweisen in der Gegenwartsliteratur von Frauen. Dülmen: Tende 1987.

${ }^{13}$ Z. B. Jane Austen, Mary Shelley, die Schwestern Brontë, George Eliot, Elisabeth Barrett Browning oder Emily Dickinson. Vgl.: Sandra Gilbert, Susan Gubar: The Madwoman in the Attic. The Woman Writer and the Nineteenth-Century Literary Imagination. New Haven, London: Yale UP 1979.

${ }^{14}$ Z. B. Sigrid Weigel: “"Wär ich ein Mann, hätte ich aus diesem Zustand vielleicht ein Werk geschaffen': Unica Zürn.” In: Inge Stephan, Regula Venske, Sigrid Weigel (Hg.): Frauenliteratur ohne Tradition? Neun Autorinnenporträts. Frankfurt a.M.: Fischer 1987, 243-277.

${ }^{15}$ Z. B. Irmela von der Lühe: "Erzählte Räume - leere Welt: zu den Romanen Marlen Haushofers." In: Anne Duden u. a.: "Oder war da manchmal noch etwas anderes?" Texte zu Marlen Haushofer. Frankfurt a.M.: Neue Kritik 1986, 73-107. Konstanze Fliedl: "Die melancholische Insel. Zum Werk Marlen Haushofers." In: Vierteljahresschrift 35 (1986), $25-52$.

${ }^{16} \mathrm{Z}$. B. Christa Gürtler: Schreiben Frauen anders? Untersuchungen zu Ingeborg Bachmann und Barbara Frischmuth. Stuttgart: Heinz 1983. Monika Albrecht: Die andere Seite: Untersuchungen zur Bedeutung von Werk und Person Max Frischs in Ingeborg Bachmanns 'Todesarten.' Würzburg: Königshausen \& Neumann 1989. Ortrud Gutjahr: 'Faschismus in der Geschlechterbeziehung? Die Angst vor dem anderen und geschlechtsspezifische Aggression in Ingeborg Bachmanns 'Der Fall Franza.'” In: Freiburger literaturpsychologische Gespräche 6 (1987), 89-100. Dies.: Fragmente unwiderstehlicher Liebe: zur Dialogstruktur literarischer Subjektentgrenzung in Ingeborg Bachmanns'Der Fall Franza.' Würzburg: Königshausen \& Neumann, 1988. Marianne Schuller: "Wider den Bedeutungswahn. 
wurden nun eingehender literaturwissenschaftlicher Analyse unterzogen. Aber auch Texte von Autoren wurden im Hinblick auf die in ihnen entworfenen Weiblichkeitsbilder in Weiterführung der Images-of-WomenForschung ${ }^{17}$ hinterfragt.

Für die Untersuchung der Projektionsmechanismen in der Konstruktion von Weiblichkeitsbildern wurde Silvia Bovenschens Buch Die imaginierte Weiblichkeit aus dem Jahre 1979 grundlegend. Mythen von Weiblichkeit in der Literatur werden als "Männerphantasien"18 gelesen, deren Wirkungsmächtigkeit die Selbstauffassungen beider Geschlechter soziokulturell geprägt habe. Bovenschen insistiert jedoch auf der Differenzqualität literarischer Weiblichkeitsentwürfe, von denen nicht unmittelbar auf die historische Realität von Frauen zurückgeschlossen werden kann. Deutlich wird an dieser Studie auch, dass die feministische Theoriebildung in Deutschland nicht nur wesentlich durch Positionen des Feminist Literary Criticism in den USA und Ansätze der Écriture féminine in Frankreich getragen ist, ${ }^{19}$ sondern auch durch eine spezifische Form der Wiederaneignung und Weiterentwicklung der psychoanalytischen Theorie. Denn in Deutschland und Österreich konnte während der Zeit des Nationalsozialismus die Psychoanalyse kaum rezipiert und weiterentwickelt werden. In den USA war die Psychoanalyse hingegen schon früh popularisiert worden und konnte mit dem Feminismus und einer feministisch orientierten Literaturwissenschaft eine selbstverständliche Allianz eingehen. Auch in Frankreich war Freuds Theorie mit neuer Ausprägung weiter entwickelt worden. Somit war der Zugang zur Psychoanalyse innerhalb der feministischen Literaturwissenschaft in Deutsch-

Zum Verfahren der Dekomposition in 'Der Fall Franza'." In: Heinz Ludwig Arnold (Hg.): Ingeborg Bachmann. München: Text und Kritik 1984,150-156.

${ }^{17}$ Kate Milletts Dissertation Sexual Politics, New York: Doubleday 1970, erschien 1971 unter dem Titel Sexus und Herrschaft. Die Tyrannei des Mannes in unserer Gesellschaft. Köln: Kiepenheuer \& Witsch 1982.

${ }^{18} \mathrm{Vgl}$. dazu auch Klaus Theweleit: Männerphantasien. Bd. 1: Frauen, Fluten, Körper, Geschichte. Frankfurt a.M.: Roter Stern 1977. - Bd. 2: Männerkörper: Zur Psychoanalyse des weißen Terrors, Frankfurt a.M.: Roter Stern 1978.

i9 Inge Stephan, Sigrid Weigel u. a. (Hg.): Feministische Literaturwissenschaft. Berlin: Argument 1984. Dies.: (Hg.): Die verborgene Frau. Berlin: Argument, 1985. Dies.: (Hg.): Weiblichkeit und Avantgarde. Berlin:Argument 1987. 
land auch durch die Theoriebildungen in den USA und Frankreich vermittelt. Diese feministisch orientierte Auseinandersetzung mit Freuds Theorie war ob ihrer Weiblichkeitskonstruktion von Anbeginn dezidiert kritisch. Denn moniert wurde, dass die Unterlegenheit der Frau nicht sozial- und ideologiekritisch als Effekt patriarchalischer Strukturen erklärt, sondern im Sinne eines psychobiologischen Mangels naturalisiert wurde. Kritisiert wurde vor allem, dass Freud seine Theorie zur Herausbildung von Geschlechtsidentität an der männlichen Entwicklung ausrichtet und davon abgeleitet weibliche Geschlechtsidentität als defizitär fasst. ${ }^{20}$

Eine kämpferische Opposition gegen Freuds Weiblichkeitskonstruktion, wie sie bereits durch Karen Horney formuliert worden war, ${ }^{21}$ erhielt durch den Feminismus als politische Bewegung neue Schubkraft. Nach dem amerikanischen psychoanalytischen Feminismus der 70er Jahre, der Freuds Theorie zur weiblichen Sexualität Phallozentrismus vorwarf, wurden in den $80 \mathrm{er}$ und 90er Jahren mit den psychoanalytisch orientierten Feministinnen Dorothy Dinnerstein, ${ }^{22}$ Nancy Chodorow, ${ }^{23}$ Carol Gilligan $^{24}$ und Jessica Benjamin ${ }^{25}$ neue Ansätze zur Mutter-Kind-Dyade und Weiblichkeit entwickelt, die auch in Deutschland innerhalb der feministischen Literaturwissenschaft breit rezipiert wurden. Tendenziell verlagerte sich nun der Schwerpunkt von der Kritik an Freuds defizitärer Weiblichkeitskonstruktion hin zu positiv besetzten Entwürfen weiblicher

\footnotetext{
${ }^{20}$ Sarah Kofman: The Enigma of Woman: Woman in Freud's Writings. Ithaca: Cornell UP 1985.

${ }_{21}$ Karen Horney: Die Psychologie der Frau. Frankfurt a.M.: Fischer 1984 [Feminine Psychology. New York: Norton 1973]. Horney war eine der ersten Analytikerinnen, die eine feministische Position gegenüber Freuds Weiblichkeitstheorie einnahm, was ihr den Ausschluss aus der psychoanalytischen Vereinigung eintrug.

${ }^{22}$ Dorothy Dinnerstein: Das Arrangement der Geschlechter, Stuttgart: Deutsche VerlagsAnstalt 1979 [The Mermaid and the Minotaur, New York: Harper \& Row 1976].

${ }^{23}$ Nancy Chodorow: Das Erbe der Mütter. Psychoanalyse und Soziologie der Geschlechter. München: Frauenoffensive 1985 [The Reproduction of Mothering: Psychoanalysis and the Sociology of Gender. Berkeley: University of California Press 1978].

${ }^{24}$ Carol Gilligan: Die andere Stimme: Lebenskonflikte und Moral der Frau. München: Piper 1984 [In a Different Voice: Psychological Theory and Women's Development. Cambridge: Harvard UP 1982].

${ }_{25}$ Jessica Benjamin: Die Fesseln der Liebe. Psychoanalyse, Feminismus und das Problem der Macht. Frankfurt a.M.: Stroemfeld 1990 [The Bonds of Love: Psychoanalysis, Feminism, and the Problem of Domination. New York: Pantheon 1988].
} 
Sexualität. Bereits 1981 hatte Renate Schlesier in ihrem Werk Konstruktionen der. Weiblichkeit bei Sigmund Freud formuliert, dass die "Weiblichkeitstheorie kein vom Ganzen der Psychoanalyse ablösbarer Teilbereich ist, dessen Grundpositionen konsequenzenlos abgewandelt oder aufgegeben werden könnten; vielmehr erweist sich diese als ein Prüfstein, an dem aufklärerischer Anspruch, innere Stimmigkeit und weiterwirkende methodologische und erkenntnistheoretische Interessen der Psychoanalyse gemessen werden können. ${ }^{, 26}$ In vergleichbarer Weise hatte Juliet Mitchell in ihrem 1985 auf Deutsch erschienenen Buch Psychoanalyse und Feminismus $^{27}$ moniert, dass im Zuge der feministischen Lektüre die Weiblichkeitskonstruktion aus dem Gesamtzusammenhang der psychoanalytischen Theorie herausgelöst worden sei. Sie sieht im Modell der Psychoanalyse gerade die Chance, das patriarchale System zu rekonstruieren und den Einfluss des Unbewussten auf die soziale Wirklichkeit zu hinterfragen.

Ganz in diesem Sinne hat Christa Rohde-Dachser Freuds Weiblichkeitskonstruktion als Prüfstein für den aufklärerischen Anspruch der Psychoanalyse untersucht. In ihrem Buch Expedition in den dunklen Kontinent aus dem Jahre 1991 wird in der Rede vom "dunklen Kontinent" ein Bereich des Unbewussten im Diskurs der Psychoanalyse ausgemacht. Rohde-Dachser verfolgt einen genuin psychoanalytischen Ansatz, insofern sie Freuds Weiblichkeitskonstruktion gleichsam auf die 'feministische Analysecouch' legt, um die Konstruktion selbst auf die ihr zugrunde liegenden unbewussten Phantasien zu untersuchen. Sie liest Freuds Weiblichkeitstheorie als "Sozialisationstheorie des Patriarchats," die zugleich die "unbewußten Prozesse" sichtbar macht, die bei der Sozialisation der Geschlechter in die patriarchalische Gesellschaft wirksam werden und dazu beitragen, dass "das für die Gesellschaft typische

\footnotetext{
${ }^{26}$ Renate Schlesier: Konstruktionen der Weiblichkeit bei Sigmund Freud: Zum Problem von Entmythologisierung und Remythologisierung in der psychoanalytischen Theorie. Frankfurt a.M.: Europ. Verl. Anst. 1990, 11.

27 Juliet Mitchell: Psychoanalyse und Feminismus. Frankfurt a.M.: Suhrkamp 1976. [Psychoanalysis and Feminism. New York: Pantheon Books 1974].
} 
Geschlechterverhältnis sich immer wieder reproduziert." ${ }^{, 28}$ Sie formuliert die Hauptpunkte von Freuds Weiblichkeitskonstruktion als in Theoriesprache gefasste Derivate unbewusster Phantasien, in denen sich zugleich kollektive unbewusste Phantasien widerspiegeln. Kulturkritisch betrachtet übernehme die Frau "Containerfunktion" für abgespaltene verdrängte männliche Anteile. Rohde-Dachser kommt bei der Analyse von Freuds Weiblichkeitskonstruktion zu dem Ergebnis, dass die Auflösung des Rätsels "Weib" einer Rücknahme der projizierten Inhalte in die männliche Selbstdefinition gleichkommen würde. Sie kommt weiter zum Schluss, dass sich das patriarchalische Geschlechterverhältnis mit seinen Mystifikationen, Asymmetrien und kollektiven, bewussten und unbewussten Phantasien mehr oder minder ungebrochen in den psychoanalytischen Theorie-Diskurs hinein fortgesetzt und dort eine Verdopplung erfahren hat. Wenn auch noch nicht unter dem Begriff Gender gefasst, so impliziert Rohde-Dachsers Ansatz bereits die Forderung, die Konstruktion von Geschlecht auf seine diskursiven Vorgaben und seine rhetorische Verfasstheit zu befragen.

Gegenüber der amerikanischen feministischen Bewegung war der französische Feminismus auf Text- und Schreibtheorien gerichtet, die sich dem Post- und Neostrukturalismus in der Nachfolge de Saussures verdanken. Theoretikerinnen und Schriftstellerinnen, die sich der Écriture féminine zurechnen lassen, wie Hélène Cixous, ${ }^{29}$ Luce Irigaray, ${ }^{30}$ Julia Kristeva, ${ }^{31}$ Chantal Chawaf ${ }^{32}$ oder Monique Wittig, ${ }^{33}$ haben die Psycho-

\footnotetext{
${ }^{28}$ Christa Rohde-Dachser: Expedition in den dunklen Kontinent. Weiblichkeit im Diskurs der Psychoanalyse. Berlin, Heidelberg 1991, 55.

${ }^{29}$ Hélène Cixous: Die unendliche Zirkulation des Begehrens. Berlin: Merve 1977.

${ }^{30}$ Luce Irigaray: Das Geschlecht das nicht eins ist. Berlin: Merve, 1979 [Ce sexe qui n'en est pas un. Paris: Éditions de minuit 1977] und Dies.: Speculum. Spiegel des anderen Geschlechts. Frankfurt a.M.: Suhrkamp 1980 [Speculum de l'autre femme. Paris: Éditions de minuit 1974].

${ }^{31}$ Julia Kristeva: Die Revolution der poetischen Sprache. Frankfurt a.M. 1978 [La révolution du langage poétique: L' Avantgarde à la fin du XIXe siècle: Lautréamont et Mallarmé. Paris : Seuil 1974].

${ }^{32}$ Chantal Chawaf: Retable. Paris : Éditions des femmes 1974 u. Dies.: Chair chaude. Paris : Mercure de France 1976.

${ }^{33}$ Monique Wittig: Aus deinen zehntausend Augen, Sappho. Berlin: Amazonen-Frauenverlag 1977 [Le corps lesbien. Paris: Éditions de minuit 1974].
} 
analyse in Verbindung mit strukturalistischen und dekonstruktivistischen Ansätzen etwa von Lévi-Strauss, Foucault, Barthes und Derrida weiter entwickelt. Vor allem aber wurde Lacans strukturale Psychoanalyse, mit der das Begehren des Subjekts als sprachlich strukturiertes untersucht wird, ${ }^{34}$ mit feministischen Ansätzen verknüpft. In diesem Zusammenhang gewann auch die Figur der Hysterikerin, die ihr Begehren inszeniert, für die feministische Theoriebildung neue Aufmerksamkeit. ${ }^{35}$

Die Untersuchung von Differenzzuweisungen erhielt durch den Dekonstruktivismus eine neue Wendung. Geschlechtlichkeit wurde nicht mehr als biologisch begründbare Wesenheit, sondern als soziokulturelle Zuschreibung verstanden. Auch dieser Ansatz wurde in Deutschland durch eine doppelte Vermittlung aufgenommen, nämlich die Rezeption der Écriture féminine in den USA als French Feminism. Im Jahre 1992 erschien der von Barbara Vinken herausgegebene Sammelband Dekonstruktiver Feminismus. Literaturwissenschaft in Amerika, in dem größtenteils Aufsätze aus der ersten Hälfte der 80er Jahre zusammengestellt sind. Dieser Theorie nach ist Geschlechterdifferenz weder biologisch noch kulturell etwas Wesentliches, sondern Effekt der Signifikationspraxis, also rhetorisch verfasst. Geschlechtlichkeit wird als Effekte sprachlicher Zuweisungen und Oppositionsbildungen verstanden. ${ }^{36}$ Die Geschlechterdifferenz erscheint so als Effekt einer rhetorisch verfassten männlichen Ordnung, während das Weibliche als differenzielles Moment in dieser Ordnung ausgemacht wird. Dekonstruktive Lektüre versteht sich als Defiguration, als Kritik vorgegebener Muster und Bedeutungszuweisungen zugunsten des verdrängten differenziellen Moments.

Als Judith Butlers Buch Gender Trouble (1990) ein Jahr nach Erscheinen unter dem Titel Das Unbehagen der Geschlechter in deutscher

\footnotetext{
${ }^{34}$ Jacques Lacan: Schriften 1. Frankfurt a.M.: Suhrkamp 1975, 84f.

${ }^{35}$ Christina von Braun: Nicht Ich. Logik, Lüge, Libido. Frankfurt a.M.: Neue Kritik 1990. Regina Schaps: Hysterie und Weiblichkeit. Wissenschaftsmythen über die Frau. Frankfurt a.M.: Campus 1992. Elisabeth Bronfen: Das verknotete Subjekt. Hysterie in der Moderne. Berlin: Volk und Welt, 1998 [The Knotted Subject. Hysteria and its Discontents. Princeton: Princeton, UP 1998].

${ }^{36}$ Barbara Vinken (Hg.): Dekonstruktiver Feminismus. Literaturwissenschaft in Amerika. Frankfurt a.M.: Suhrkamp 1992.
} 
Sprache veröffentlicht wurde, war durch die hier nur kursorisch dargestellte Entwicklung von den Frauenstudien zum dekonstruktiven Feminismus das Feld für die Lektüre dieses Ansatzes bereitet. ${ }^{37}$ Butlers Buch avancierte rasch zu einem Grundlagentext in der deutschen Debatte, denn er erschien gerade zum richtigen Zeitpunkt: just als die kulturwissenschaftliche Wende einsetzte und die Auseinandersetzung mit der Kategorie Geschlecht einer kritischen Revision unterzogen wurde. ${ }^{38}$ Die in Butlers Buch reflektierte Debatte um geschlechtliche Identität verdankt sich Impulsen aus der feministischen Literaturwissenschaft, nämlich der Psychoanalyse und ihren Verbindungen mit der Linguistik, dem französischen Dekonstruktivismus, Foucaults Diskurstheorie und soziologischen Interaktionsmodellen. Essentielle Geschlechtsidentitäten werden als Effekte kultureller Normen lesbar gemacht und jeglicher Rekurs auf vordiskursive Geschlechterkörper als Illusion markiert. Butlers Thesen stellten die feministische Literaturwissenschaft, wie sie sich in den 60er und 70er Jahren mit emanzipatorischem Selbstverständnis herausgebildet hatte, in Frage. Die Untersuchung von Weiblichkeitsbildern in der Literatur als Ausdruck männlicher Projektionen schien nun obsolet. Die große Wirkung, die das Buch hatte, verdankt sich dem Ansatz, Geschlechterbeziehungen "nicht länger als Ausdruck oder Repräsentation einer statischen, naturgegebenen Ordnung" zu sehen, sondern als "Repräsentationen von kulturellen Regelsystemen.",39

Butler stellt jegliche Form von Naturalismus und Essentialismus wie auch die Logik binärer Strukturen und die heterosexuelle Matrix als gesellschaftliches Organisationsprinzip in Frage. Sie geht davon aus, dass das biologische Geschlecht (sex) niemals als natürliches Geschlecht,

\footnotetext{
${ }^{37}$ Judith Butler: Das Unbehagen der Geschlechter. Frankfurt a.M.: Suhrkamp 1991 [Gender Trouble: Feminism and the Subversion of Identity. New York: Routledge 1990.

${ }^{38}$ Zur Rezeption von Butlers Buch: Isabell Lorey: Immer Ärger mit dem Subjekt. Theoretische und politische Konsequenzen eines juridischen Machtmodells: Judith Butler. Tübingen: Ed. Diskord. 1996. Christine Hauskeller: Das paradoxe Subjekt. Unterwerfung und Widerstand bei Judith Butler und Michel Foucault. Tübingen: Ed. Diskord. 2000. Karin Ludewig: Die Wiederkehr der Lust. Körperpolitik nach Foucault und Butler. Frankfurt a.M.: Campus 2002 und Feministische Studien: Kritik der Kategorie Geschlecht 11.2 (1993).

${ }^{39}$ Renate Hof: Die Grammatik der Geschlechter, "Gender" als Analysekategorie der Literaturwissenschaft. Frankfurt a.M., New York: Campus 1995, 16.
} 
sondern nur über soziokulturelle Vermittlung überhaupt wahrgenommen werden kann. Auch Butler kritisiert die Psychoanalyse, da hier Normen formuliert seien, die einer heterosexuellen Matrix folgen..$^{40}$ Demgegenüber fragt sie nach den diskursiven Verfahren, welche Geschlechtsidentität konstituieren. Präferiert werden bei ihr gender acts, also beispielsweise parodistische Praktiken, mit denen Kategorien von Geschlecht und Sexualität ins Wanken gebracht werden. Butler versteht Vergeschlechtlichung als fortwährende gender performances, die darin bestehen, dass kulturelle Zuschreibungen bewusst am eigenen Körper wiederholt werden, damit sie als solche erkannt werden können. Parodie und Travestie sollen sichtbar machen, dass Geschlechtsidentität nichts anderes als eine performative Aneignung von Zuschreibungen sei. Konzepte der Maskerade, wie sie Butler in Gender Troubles in Anknüpfung an Rivière, ${ }^{41}$ Lacan und dem poststrukturalistischen Feminismus entwickelt hatte, wurden auch in der Genderdebatte in Deutschland weiter entwickelt. ${ }^{42}$ Maskerade wird als Nachahmung eines nicht vorhandenen Originals verstanden $^{43}$ und Geschlecht als Kompromissbildung, mit der Konflikte und Widersprüche verhüllt und zugleich bewahrt bleiben.

In der Rezeption von Butlers Buch in Deutschland wurde vor allem die Verdrängung des Leibes aus der Geschlechtskonstruktion moniert. ${ }^{44}$

\footnotetext{
${ }^{40}$ Der Genderdiskurs der 90er Jahre hat klassische feministische Grundannahmen wie Frau, Identität und Subjekt unterwandert und dekonstruiert. Teresa de Lauretis hat in ihrer Studie The Practice of Love: Lesbian Sexuality and Perverse Desire. Bloomington: Indiana UP 1994, die 1996 unter dem Titel Die andere Szene. Psychoanalyse und lesbische Sexualität, Berlin: Berlin-Verlag 1996 ins Deutsche übersetzt wurde, in Auseinandersetzung mit der Psychoanalyse Sexualität als semiotischen Prozess betrachtet.

4! Joan Rivière: "Weiblichkeit als Maskerade." In: Liliane Weissberg (Hg.): Weiblichkeit als Maskerade, Frankfurt a.M.: Fischer 1994, 34-47 [zuerst in engl. veröffentlicht als "Womanliness as a Masquerade." In: The International Journal of Psychoanalysis 10 (1929), 3644].

${ }^{42}$ Elfi Bettinger, Julika Funk (Hg.): Maskeraden. Geschlechterdifferenz in der literarischen Inszenierung. Berlin: Erich Schmidt 1995.

${ }^{43}$ Vgl. hierzu auch: Gertrud Lehnert: Wenn Frauen Männerkleider tragen. Geschlecht und Maskerade in Literatur und Geschichte. München: DTV 1997.

${ }^{44}$ Benhabib kritisierte vor allem den Subjektbegriff und Lindemann die Verdrängung des Körpers bei Butler. Hierzu: Seyla Benhabib: "Feminismus und Postmoderne." In: Dies.: Judith Butler, Drucilla Cornell, Nancy Fraser (Hg.): Der Streit um Differenz. Feminismus und Postmoderne in der Gegenwart. Frankfurt a.M.: Fischer 1993, 9 und Gesa Lindemann: "Wider die Verdrängung des Leibes aus der Geschlechterkonstruktion." In: Feministische
} 
Beklagt wurde auch, dass Butler die Zeichenhaftigkeit des Körpers thematisiert, aber Generativität ausblendet. ${ }^{45}$ Denn innerhalb der Gender Studies kommt dem Körper als Medium der Einschreibung kultureller Verwerfungen eine herausgehobene Position zu. ${ }^{46}$ In Fortführung der früheren Hysterieanalysen kann beispielsweise die Präsentation des weiblichen Körpers als kulturelle performance verstanden werden. ${ }^{47}$ Aus psychoanalytischer Perspektive hat Reimut Reiche sich intensiv mit Geschichte und Funktionswandel des Begriffs Gender auseinander gesetzt und herausgestellt, dass mit dieser Übernahme des Begriffs in die Theoriebildung in Deutschland Freuds Konzept der Bisexualität verdrängt worden sei. ${ }^{48}$ Reiche geht davon aus, dass die Trope gender kreiert wurde, um die Abstoßung von der Trope sex in der Geschlechtsidentität zu markieren. Er wendet sich gegen die Einführung des Konzepts der "core gender identity" und insistiert darauf, dass sich Geschlechtsidentität und geschlechtliche Orientierung nicht von einander trennen lassen. Er beruft sich auf interdisziplinäre Studien, die davon ausgehen, dass die biologische Zweigeschlechtlichkeit und das soziale Geschlecht miteinander verbunden sind und dass die Zuweisung von Männlichkeit und Weiblichkeit vielfach bedingt ist.

Mit der Übernahme des Begriffs Gender in den deutschen Sprachgebrauch wird noch einmal deutlich, dass die Entwicklung der feministischen Theorie ohne den Transfer aus den USA undenkbar ist. Der deutsche Begriff Geschlecht wird damit aber nicht obsolet, denn er umfasst

Studien 2 (1993), 44-54. Siehe auch die anderen Diskussionsbeiträge in Feministische Studien 2 (1993).

${ }^{45}$ Vgl. hierzu: Andrea Maihofer: Geschlecht als Existenzweise: Macht, Moral, Recht und Geschlechterdifferenz. Frankfurt a.M.: Helmer 1997.

${ }^{46}$ Doris Bischof-Köhler: Von Natur aus anders: die Psychologie der Geschlechtsunterschiede. Stuttgart: Kohlhammer 2002.

${ }^{47}$ Vgl. hierzu auch: Bettina Pohle: Kunstwerk Frau. Inszenierungen von Weiblichkeit in der Moderne Frankfurt a.M.: Fischer 1998. Auch Thomas Laqueur hat mit seinem Buch Auf dem Leib geschrieben. Die Inszenierung der Geschlechter von der Antike bis Freud. Frankfurt a.M.: Campus, 1992 [Making Sex: Body and Gender from the Greeks to Freud. Cambridge: Harvard UP 1990] herausgearbeitet, dass im 18. Jahrhundert mit der Veränderung diskursiver Praktiken ein neuer Begriff von Gender entstanden sei.

${ }^{48}$ Reimut Reiche: "Gender ohne Sex. Geschichte, Funktion und Funktionswandel des Begriffs 'Gender."' In: Psyche 51 (1997), 927-958. 
ein weites semantisches Feld, das sich der Erschließung lohnt. Der lateinische Begriff Genus, von dem sich Geschlecht ableitet, meint Geburt, Abkunft, Stamm, Haus, Familie, Nachkomme, Gattung und Klasse. In der ursprünglichen Bedeutung bezeichnete Geschlecht die Art des Menschen (das Menschengeschlecht), wie auch die blutsverwandte Familie oder Sippe, die auf einen Stammvater zurückgeht. ${ }^{49}$ Im Weiteren werden aber auch die sekundären Geschlechtsmerkmale eines Menschen sowie seine Zugehörigkeit zu einer der dichotom bestimmten Gruppen von Männern oder Frauen damit gemeint. Der Begriff Geschlecht umspannt also einerseits sowohl eine Einheit (Menschheit) wie auch eine Spaltung (Männer und Frauen). Andererseits bezieht sich Geschlecht auf biologische wie soziokulturelle Differenzbestimmungen, die durch diskursive Vorgaben geprägt sind. ${ }^{50}$ Komposita erweitern und präzisieren dieses semantische Feld von Geschlecht noch weiter. So wird etwa mit dem Begriff Geschlechtsidentität das durch Sozialisation in einer Kulturgemeinschaft erworbene Bewusstsein gefasst, einem Geschlecht anzugehören, das sich vom anderen Geschlecht abgrenzt. Geschlechtsidentität meint somit auch die Selbstwahrnehmung eines Subjekts als geschlechtlich bestimmtes. Der Begriff Geschlechtscharakter verweist demauf eine Festschreibung von Eigenschaften und Verhaltensweisen, die für einen Zeitraum gleichsam geschlechtsspezifisch kanonisiert werden. Hierbei handelt es sich um eine diskursive Konstruktion von dichotomen Zuordnungen. Mit dem Begriff Geschlechterrolle wird in Anlehnung an die soziologische Rollentheorie ein gesellschaftlich erwartetes Verhalten gefasst, durch das eine weibliche oder eine männliche Position zu erkennen gegeben wird. Unter Geschlechterrolle kann somit das soziale Verhalten gemäß einer Geschlechterbestimmung verstanden werden, wie auch ein spielerischer Umgang mit diesen Geschlechtszuschreibungen. Mit dem Begriff Geschlechterverhältnisse werden die Beziehungen der Geschlechter untereinander nach Maßgabe soziokultureller Vorgaben und

\footnotetext{
49 Jacob und Wilhelm Grimm: Deutsches Wörterbuch, 33 Bde., München: DTV 1984, Bd. 5, $3903 \mathrm{ff}$.

${ }^{50} \mathrm{Vgl}$. hierzu auch die entsprechenden Einträge in: Renate Kroll (Hg.): Metzler-Lexikon Gender Studies, Geschlechterforschung: Ansätze-Personen-Grundbegriffe. Stuttgart: Mètzler 2002 .
} 
unter den Bedingungen epochenspezifischer Geschlechterdiskurse bestimmt.

Obwohl der Begriff Genus (Verb: genere = erzeugen, erschaffen, hervorbringen) auf die Erzeugung von Bedeutungen, die Herstellung von Klassifikationen und die Konstruktion von Beziehungen hinweist, konnte er sich innerhalb der deutschsprachigen Geschlechterforschung nicht etablieren. ${ }^{51}$ Genus wird im deutschen Sprachgebrauch als lexikalischgrammatische Kategorie verwendet. In der Literatur- und Kunstwissenschaft dient der Begriff genre (der französische Begriff für Gender) auch der Kategorisierung innerhalb von Gattungen oder Gebieten. Im deutschsprachigen Zusammenhang wird Gender im Sinne von soziokulturell konstruiertem Geschlecht im Gegensatz zum biologischen Geschlecht gebraucht. Der englisch-amerikanische Gender-Begriff konnte sich im deutschsprachigen Sprachgebrauch etablieren, weil er theoretisch wesentlich stärker eingebunden ist als der Begriff Geschlecht. Populär wurde die Kategorie Gender aber vor allem durch die deutsche Übersetzung von Butlers Gender Trouble, denn keine theoretische Debatte hat die feministische Forschung so polarisiert und zugleich revidiert wie ihr Buch.

Gender Studies sind mittlerweile nicht mehr marginales Randgebiet innerhalb der Wissenschaften, sondern diskussionsbeherrschend. Die feministische Wissenschaftskritik wurde institutionell verankert und $\mathrm{zu}$ einem Forschungsfeld, das mehr und mehr Ansehen genießt. Professuren wurden zu diesem Schwerpunkt ausgeschrieben und Graduiertenkollegs zu diesem Themengebiet ermöglichten eine Nachwuchsförderung, mit welcher der Genderforschung immer breiterer Raum eingeräumt wurde. ${ }^{52}$

${ }^{51}$ Ein Versuch, die Kategorie Genus zu etablieren, wurde unternommen mit dem Band: Renate Hof, Hadumod Bußmann (Hg.): Genus. Zur Geschlechterdifferenz in den Kulturwissenschaften. Stuttgart: Kröner 1995.

52 Besonders einflussreich war das Münchener Graduiertenkolleg "Geschlechterdifferenz und Literatur," das seit Anfang der 90er Jahre gefördert wurde. Im Rahmen dieses Graduiertenkollegs wurden zahlreiche Symposien veranstaltet und Sammelbände publiziert. Beispiele hierfür sind Ina Schabert, Barbara Schaff (Hg.): Autorschaft: Genus und Genie in der Zeit um 1800. Berlin: Erich Schmidt 1994. Birgit Wägenbaur: Die Pathologie der Liebe: literarische Weiblichkeitsentwürfe um 1800. Berlin: Erich Schmidt, 1996. Claudia Öh1schläger (Hg.): Körper-Gedächtnis-Schrift: der Körper als Medium kultureller Erinnerung. Berlin: Erich Schmidt, 1997. Kati Röttger (Hg.): Differenzen in der Geschlechterdifferenz: 
In eigens eingerichteten Reihen wurden die Ergebnisse dieser Forschungen publiziert. ${ }^{53}$ Ebenso sind zahlreiche Zeitschriften zur feministischen Forschung und zu Genderstudien erschienen. ${ }^{54}$ An vielen deutschen Universitäten können mittlerweile Gender Studies zumeist in transdisziplinärer Zusammenarbeit von Kultur-, Sprach-, Literatur- und Sozialwissenschaften studiert werden und Zentren für interdisziplinäre Frauenund Geschlechterforschung wurden etabliert.

Die Gender Studies können als Grundlage, als Fortsetzung oder Überwindung der feministischen Literaturwissenschaft gesehen werden. Durch sie kam es zu einer Ausdifferenzierung der Geschlechterthematik und einer Verknüpfung mit anderen Kategorien gesellschaftlicher Differenzierung. So werden innerhalb der Gender Studies auch andere Differenzstudien wie Queer Studies, ${ }^{55}$ interkulturelle ${ }^{56}$ oder postkoloniale ${ }^{57}$ Studien betrieben und die Konstruktion von Männlichkeit rückt ins Blickfeld des

aktuelle Perspektiven der Geschlechterforschung. Berlin: Erich Schmidt 1999 in der Reihe "Geschlechterdifferenz und Literatur."

${ }^{53}$ Die von Ina Schabert und Gerhard Neumann herausgegebene Reihe "Geschlechterdifferenz und Literatur" im Erich Schmidt-Verlag und die von Inge Stephan und Sigrid Weigel herausgegebene Reihe Literatur-Kultur-Geschlecht im Böhlau-Verlag sowie die Reihe Gender Studies im Suhrkamp Verlag.

${ }^{54}$ Rundbrief Frauen in der Literaturwissenschaft, der von der Hamburger Arbeitsstelle für feministische Literaturwissenschaft herausgegeben wurde. Die Zeitschriften Metis. Zeitschrift für historische Frauenforschung und feministische Praxis (Dortmund). Die Philosophin. Forum für feministische Theorie und Philosophie (Tübingen). Querelles. Jahrbuch für Frauenforschung (Göttingen). Feministische Studien (Stuttgart). Figurationen: Gender, Literatur, Kultur (Köln).

${ }^{55}$ Gesa Lindemann: Das paradoxe Geschlecht. Frankfurt a.M.: Fischer 1993. Alexandra Busch, Dirck Linck: Frauenliebe/Männerliebe. Eine lesbisch-schwule Literaturgeschichte in Porträts. Ștuttgart: Metzler 1997. Ulf Heidel: Jenseits der Geschlechtergrenzen: Sexualitäten, Identitäten und Körper in Perspektiven von Queer Studies. Hamburg: MännerschwarmSkript 2001. Andreas $\mathrm{Kraß}$ (Hg.): Queer denken: gegen die Ordnung der Sexualität. Frankfurt a.M.: Suhrkamp 2003.

${ }^{56} \mathrm{Z}$. B. Judith Schlehe (Hg.): Zwischen den Kulturen - zwischen den Geschlechtern. Münster: Waxmann 2000. Judith Schlehe (Hg.): Interkulturelle Geschlechterforschung: Identitäten - Imaginationen - Repräsentationen. Frankfurt a. M.: Campus 2001.

${ }^{57}$ Hito Steyerl (Hg.): Spricht die Subalterne deutsch?: Migration und postkoloniale Kritik. Münster: Unrast 2003. Encarnacíon Gutíerrez Rodríguez: "Fallstricke des Feminismus. Das Denken 'kritischer Differenzen' ohne geopolitische Kontextualisierung. Einige Überlegungen zur Rezeption antirassistischer und postkolonialer Kritik." In: polylog. Zeitschrift für

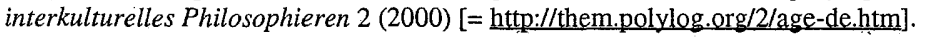


Interesses. ${ }^{58}$ Zentrale Frage wird nun, wie die Untersuchung von Geschlecht mit anderen Differenzanalysen verzahnt werden kann. ${ }^{59}$ Denn Gender-Studien untersuchen, wie solche Differenzzuschreibungen in verschiedenen Zusammenhängen und Epochendiskursen hergestellt werden und welche Bedeutung ihnen beigemessen wird. Mit dem Begriff Gender wird nach der Funktion und den Konsequenzen von Differenzierungen, Polarisierungen und Hierarchisierungen gefragt. Der ehemals feministische Ansatz wechselt damit auch in eine interdisziplinär ausgerichtete Kulturwissenschaft, die sich nun prinzipiell mit der Konstruktion von Geschlecht auch in wissenschaftlichen Argumentationsmustern auseinander setzt. $^{60}$ Aufgehoben ist damit auch eine Dichotomisierung zwischen Frauenforschung und kanonisierter Wissenschaft zugunsten einer Etablierung der Kategorie Geschlecht in den kulturwissenschaftlichen Forschungsbereich. ${ }^{61}$ Die Gender-Studien verstehen sich aber auch als eine Form der Wissenschaftskritik, insofern Konstruktionsbedingungen des Faches und des Wissenskanons hinterfragt werden.

\footnotetext{
${ }^{58}$ Z. B. Stefan Beier: Kritische Männerforschung: neue Ansätze in der Geschlechtertheorie. Berlin: Argument 1996. Constance Engelfried: Männlichkeiten: die Öffnung des feministischen Blicks auf den Mann. Weinheim: Juventa 1997. Doris Janshen: Blickwechsel: der neue Dialog zwischen Frauen- und Männerforschung. Frankfurt a.M.: Campus, 2001. Ursula Pasero, Christine Weinbach (Hg.): Frauen, Männer, Gender trouble: systemtheoretische Essays. Frankfurt a.M.: Suhrkamp 2003.

${ }^{59}$ Z. B. in Brigitte Rommelspacher: Dominanzkultur: Texte zu Fremdheit und Macht. Berlin: Orlanda Frauenverl. 1995. Sedef Gümen: "Die sozialpolitische Konstruktion 'kultureller' Differenzen in der bundesdeutschen Frauen- und Migrationsforschung." In: Beiträge zur feministischen Theorie und Praxis 42 (1996),77-90. Sabine Schülting: Wilde Frauen, fremde Welten. Kolonisierungsgeschichten aus Amerika. Reinbek bei Hamburg: Rowohlt 1997. Heidi Armbruster: "Feministische Theorien und Ethnologie." In: polylog. Zeitschrift für interkulturelles Philosophieren 4 (1999) [http://lit.polylog.org/2/eah-de.htm]. Claudia Rademacher, Peter Wiechens (Hg.): Geschlecht, Ethnizität, Klasse. Zur sozialen Konstruktion von Hierarchie und Differenz. Opladen: Leske \& Budrich 2001. Birgit Sauer: "Ethnizität (Ethnozentrismus)." In: Renate Kroll (Hg.): Metzler-Lexikon Gender Studies, Geschlechterforschung: Ansätze - Personen - Grundbegriffe, Stuttgart: Metzler 2002, 92f.

${ }^{60}$ Von Braun und Stephan plädieren für eine Beibehaltung der feministischen Forschung neben der genderorientierten Forschung. Christina von Braun, Inge Stephan (Hg.): GenderStudien: Eine Einfuihrung, Stuttgart: Metzler 2000, 11.

${ }^{61}$ Dörte Bischoff: "Gender-Theorien. Neuere deutsche Literatur." In: Claudia Benthien, Hans Rudolf Velten (Hg.): Germanistik als Kulturwissenschaft. Eine Einführung in neue Theoriekonzepte. Reinbek bei Hamburg: Rowohlt 2002, 298-322.
} 
Es geht mithin also um die Art und Weise, wie über kulturspezifische Bedeutungszuschreibungen Differenzen generiert, Dichotomisierungen eingeführt und Hierarchien produziert werden. Der Begriff Gender ist somit aber auch in die gegenwärtigen Subjekt- und Identitätsdiskurse eingebettet. Dabei erweist sich Fremde als zentrale Analysekategorie. Wie es Renate Hof in ihrer Studie Die Grammatik der Geschlechter aus dem Jahre 1995 formuliert hat, geht es um eine "Auseinandersetzung mit der Fremdheit des anderen und des eigenen Geschlechts." ${ }^{62}$ Die Bedeutungszuschreibungen aber, die für die Konstruktion von Fremde kennzeichnend sind, wurden durch die Differenzanalysen der Interkulturalitätsforschung herausgearbeitet.

Interkulturalität ist nicht nur zu einem intensiv umworbenen Konzept innerhalb wissenschaftlicher Diskurse geworden, sondern auch zu einem zentralen Begriff innerhalb der öffentlichen Diskussion. ${ }^{63}$ Seine Vielschichtigkeit und den damit verbundenen hohen Attraktionswert gewinnt das Forschungsparadigma Interkulturalität, weil es auch im bildungs-, wirtschafts- und kulturpolitischen Bereich hoch im Kurs steht. Gewinnt ein Begriff aber eine so zentrale Strukturierungsfunktion für unterschiedliche Diskursformationen, so weist dies darauf hin, dass sich gesellschaftliche Verschiebungen und Neuorientierungen in ihm niederschlagen. Interkulturalität ist somit auch als Ausdruck einer gesellschaftsspezifischen Aufmerksamkeitsverlagerung im Zeichen eines neuen Wissens- und Erklärungsbedarfs zu verstehen.

Die erste Professur für Literaturwissenschaft/interkulturelle Germanistik wurde im Frühjahr 1986 durch die Fakultät für Geistes- und Sozialwissenschaften der Universität Karlsruhe ausgeschrieben, wo zwei

\footnotetext{
${ }^{62}$ Hof, Die Grammatik der Geschlechter, 122.

$63 \mathrm{Vgl}$. zu diesen Ausführungen auch meine Beiträge: "Interkulturalität und Interdisziplinarität." In: Arlette Bothorel-Witz, Christine Maillard (Hg.): Du Dialogue des Disciplines. Germanistique et interdisdisciplinarité, Strasbourg: Presses Univ. de Strasbourg 1998, 135-149. "Alterität und Interkulturalität." In: Claudia Benthien, Hans Rudolf Velten (Hg.): Germanistik als Kulturwissenschaft. Eine Einfihrung in neue Theoriekonzepte. Reinbek bei Hamburg: Rowohlt 2002, 345-369. Vorwort zur Sektion "Literaturwissenschaft als Kulturwissenschaft" und Einleitung zur Teilsektion "Interkulturalität und Alterität." In: $A k$ ten des X. Internationalen Germanistenkongresses Wien 2000, Bd. 9, Bern u. a. 2003, 11-20.
} 
Jahre zuvor auf der vierten internationalen Sommerkonferenz Deutsch als Fremdsprache auch die Gesellschaft für interkulturelle Germanistik (GiG) gegründet worden war. Es waren zunächst vor allem Lehrende aus dem Fach Deutsch als Fremdsprache, die versuchten, gemeinsam mit den Lehrenden der so genannten Inlandsgermanistik in einem für das Fach Germanistik intradisziplinären Dialog, eine neue Richtung literaturwissenschaftlicher Forschung mit kulturwissenschaftlicher Ausrichtung zu etablieren. Ziel dieser Neugründung war es, dass die Lehrenden beider Teilbereiche in Forschung und Lehre sowie in der Entwicklung von Fragestellungen und Methoden kooperativ zusammenarbeiten. ${ }^{64}$ Von den Gründungsmitgliedern wurde die interkulturelle Germanistik als eine Wissenschaft verstanden, die "von der Kultureingebundenheit aller germanistischen Arbeit in Forschung und Lehre ausgeht." ${ }^{65}$ Das ambitionierte Vorhaben, Literatur im interkulturellen Kontext zu untersuchen, überfordert jedoch Kapazität und Möglichkeit eines Einzelnen. Mit den Tagungen und Publikationen der Gesellschaft für interkulturelle Germanistik wurde ein Forum geschaffen, bei dem die unterschiedlichen Erfahrungen der im fremdsprachigen Ausland und der in den deutschsprachigen Ländern Lehrenden und Forschenden des Faches Germanistik unter fachkritischer Perspektive diskutiert werden.

Zugleich ist aber damit auch ein prinzipieller Wandel im Selbstverständnis der Germanistik angezeigt. Aufgekündigt wird nämlich die Vorstellung, dass in der Inlandsgermanistik die wissenschaftlichen Maßstäbe für die Forschung und Lehre des Faches gesetzt werden und diese dann unter Maßgabe der jeweils spezifischen kulturellen Gegebenheiten im Ausland lediglich noch modifiziert werden müssen. Vielmehr sucht die Etablierung dieser Forschungsrichtung der Veränderung Rechnung zu tragen, dass sich unter der Perspektive unterschiedlicher kultureller Kontexte nicht nur die Studentenschaft verändert hat, die an deutschen Hochschulen Germanistik studiert, sondern auch die zeitgenössische deutsch-

\footnotetext{
${ }^{64}$ Vgl. u. a.: Alois Wierlacher: Architektur interkultureller Germanistik. München: Iudicium 2001.

${ }^{65}$ Alois Wierlacher: "Einführung." In: Ders. (Hg.): Perspektiven und Verfahren interkultureller Germanistik. Akten des 1. Kongresses der Gesellschaft für interkulturelle Germanistik. München: Iudicium 1987, 13-17, hier: 13.
} 
sprachige Literatur. Die seit dem Ende der siebziger Jahre in größerer Zahl erschienenen Publikationen von Autorinnen und Autoren, die aus anderen Kulturräumen nach Deutschland immigriert sind und den Suchbewegungen zwischen den unterschiedlichen kulturellen Identifikationsangeboten eine literarische Form gaben, lenkten die Aufmerksamkeit nachhaltig auf die Gestaltung von Interkulturalität als Themengebiet und ästhetischem Verfahren in den deutschsprachigen Literaturen. ${ }^{66}$ Kulturspezifische Vermittlungs- und Rezeptionsprobleme von Literatur oder aber genuin interkulturelle Fragestellungen, wie die nach einer interkulturellen Hermeneutik, fanden in der Teildisziplin interkulturelle Germanistik ihren wissenschaftslogischen Ort, wurden aber erst damit auch explizit zum erklärten Bestandteil des Faches Germanistik insgesamt. ${ }^{67}$

Ursprünglich also aus dem Bedürfnis entstanden, den Bereich Deutsch als Fremdsprache mit der traditionellen (Inlands-)Germanistik enger zu verzahnen und ihr den Status einer eigenständigen Disziplin zu verleihen, hat die interkulturelle Germanistik im Zuge der kulturwissenschaftlichen Neuorientierung der Literaturwissenschaft einen anderen Stellenwert gewonnen und wurde als Schwerpunkt interkulturelle Literaturwissenschaft im Fach Germanistik etabliert. Im Spektrum der zunehmenden Auffächerungen innerhalb der Germanistik befindet sich nun die interkulturelle Literaturwissenschaft in der Situation, dass sie zwar einerseits auf die Forschungsergebnisse der aus dem DaF-Bereich hervorgegangenen interkulturellen Germanistik aufbauen kann, andererseits aber dieser Teildisziplin innerhalb einer Disziplinen und Fakultäten übergreifenden Diskussion um kulturwissenschaftliche Neuorientierung der Geisteswissenschaften (und insbesondere der Philologien) ein eigenständiges Gepräge geben muss. Die Begriffe interkulturelle Germanistik und

\footnotetext{
${ }^{66}$ Vgl. Mary Howard (Hg.): Interkulturelle Konfigurationen: zur deutschsprachigen Erzählliteratur von Autoren nichtdeutscher Herkunft. München: Iudicium 1997. Carmine Chiellino: Am Ufer der Fremde. Literatur und Arbeitsmigration 1870-1991. Stuttgart: Metzler 1995. Ders. (Hg.): Interkulturelle Literatur in Deutschland: ein Handbuch. Stuttgart: Metzler, 2000. Aglaia Blioumi: Interkulturalität als Dynamik: ein Beitrag zur deutsch-griechischen Migrationsliteratur seit den siebziger Jahren. Tübingen: Stauffenburg 2001.

${ }^{67}$ Alois Wierlacher: "Zur Entwicklungsgeschichte und Systematik interkultureller Germanistik (1984-1994). Einige Antworten auf die Frage: Was heißt interkulturelle Germanistik?" In: Jahrbuch Deutsch als Fremdsprache 20 (1994), 37-56.
} 
interkulturelle Literaturwissenschaft werden synonym verwandt, wobei interkulturelle Germanistik eher mit der Herkunft aus dem Bereich Deutsch als Fremdsprache verknüpft wird und interkulturelle Literaturwissenschaft mit der kulturwissenschaftlichen Wende in den Geisteswissenschaften konnotiert ist. Das Fach befindet sich also in einem produktiven Prozess der Selbstfindung und Konsolidierung und ist auch damit in die gegenwärtige Diskussion um die Kulturwissenschaften eingebettet.

Die Veränderungen, die mit dem Begriff Interkulturalität gefasst werden, beziehen sich auf neu entstandene oder verändert erlebte Formen des Austausches zwischen sozialen Einheiten und Individuen. Wenn aber tradierte Grenzziehungen aufgebrochen und zu Schnittstellen oder Kontaktflächen werden, müssen unterschiedliche Erfahrungshorizonte und Wertvorstellungen neu austariert werden. Diese für die Kommunikation zwischen sozialen Einheiten konstitutive Unterschiedlichkeit wird nun aber erst dann als kulturspezifisch wahrgenommen, wenn sie von den Kommunikationspartnern auf die Sozialisierung durch unterschiedliche Kulturen zurückgeführt und als fremd gegenüber dem eigenen System bewertet wird. Mit dem Begriff Interkulturalität werden somit Interaktionsformen bezeichnet, bei denen die Interaktionspartner sich wechselseitig als unterschiedlich kulturell geprägt identifizieren.

In ein neues Spannungsverhältnis geraten diese Interaktionsformen, wenn Nähe zwischen sozialen Gruppen und Individuen hergestellt wird, die zuvor in größerer geographischer und sozialer Distanz gelebt haben. Interkulturalität bezeichnet unter diesem sozial-räumlichen Aspekt ein Beziehungsverhältnis, das durch Nähe intensiviert und dadurch potentiell konflikthaft aufgeladen wird. Zugleich aber eignet diesem kulturdifferenten Beziehungsverhältnis auch eine temporale Komponente, insofern nicht nur Distanz verringert wird, sondern in der wechselseitigen Wahrnehmung auch unterschiedliche Zeithorizonte und Entwicklungsstadien 
aufeinander treffen und im Sinne einer Gleichzeitigkeit von Ungleichzeitigem in der Interaktion miteinander verknüpft werden müssen. ${ }^{68}$

Im Gegensatz zu den Bewegungsrichtungen der Eroberungs- und Kolonisierungsepochen, in denen seit der frühen Neuzeit weitgehend die Europäer in ferne Länder aufbrachen und dort in Kontakt mit anderen Kulturen traten, haben einerseits technisch-medialer und wirtschaftlicher Globalisierungsschub in der westlichen Welt, andererseits Arbeitsmigration und Asylsuche in den letzten Jahrzehnten des 20. Jahrhunderts dazu geführt, dass die Kontakte zwischen Menschen, die in unterschiedlichen kulturellen Kontexten sozialisiert wurden, zugenommen haben, und vielfach auf europäischem Boden stattfinden. ${ }^{69}$ Nun gehört innerhalb multikultureller Gesellschaften die Fähigkeit, sich mit unterschiedlichen kulturellen Symbolformen und Erwartungshorizonten auseinander zu setzen, zur notwendigen sozialen Kompetenz ihrer Mitglieder. ${ }^{70}$ Wie in anderen Staaten wurden auch in Deutschland Zuwanderung und deren soziale Folgen zur politischen Herausforderung und führten zu einer Verschiebung von der Gastarbeiter- und Ausländerpolitik zu einer Minderheitenund Zuwanderungspolitik. ${ }^{71}$ In diesem Zusammenhang entstand eine Nachfrage nach Integrationsmodellen auch im Sinne interkultureller Bildung und Ausbildung an den Hochschulen, was sich in gezielter Forschungsförderung zu diesen Arbeitsfeldern niederschlug.

Das verstärkt einsetzende Interesse, Interkulturalität in die universitäre Lehre und Forschung zu verankern, verdankt sich also einerseits dem Wissens- und Umorientierungsbedarf einer multikulturell geprägten Gesellschaft, andererseits aber auch den bildungspolitischen Trends, die sich

\footnotetext{
${ }^{68}$ Ortfried Schäffter: "Modi des Fremderlebens. Deutungsmuster im Umgang mit Fremdheit." In: Ders. (Hg.): Das Fremde. Erfahrungsmöglichkeiten zwischen Faszination und Bedrohung. Opladen: Westd. Verl. 1991, 11-44, hier: 12.

${ }^{69}$ Vgl. hierzu: Seyla Benhabib: "'Nous' et 'les Autres.' The Politics of Complex Cultural Dialogue in a Global Civilization." In: Christian Joppke, Steven Lukes (Hg.): Multicultural Questions. New York: Oxford UP 1999, 44-64.

${ }^{70}$ Kay Hailbronner u. a. (Hg.): Multikulturelle Gesellschaft und Wertegesellschaft. Heidelberg: Müller 2000.

${ }^{7}$ Vgl. Axel Schulte: Zwischen Diskriminierung und Demokratisierung. Aufsätze zu Politiken der Migration, Integration und Multikulturalität in Westeuropa. Frankfurt a.M.: IKOVerl. für interkulturelle Kommunikation 2000.
} 
in Deutschland und mit unterschiedlicher Vehemenz weltweit abzeichnen. Wie nie zuvor in ihrer jahrhundertelangen Geschichte ist die deutsche Universität auf Interkulturalität ausgerichtet, versucht sie sich angesichts zunehmender Globalisierung und immer knapper werdender finanzieller Ressourcen ein international anschlussfähiges wissenschaftliches Profil zu geben.

Nun koinzidiert das Interesse an Interkulturalität nicht nur mit Internationalisierungstrends infolge des Globalisierungsschubs, sondern auch unübersehbar mit der deutschen Wiedervereinigung. Fragen nach den Berührungspunkten einer gemeinsamen und doch unterschiedlichen Kultur wurden virulent. Die heftig entfachten Literaturdebatten nach der Wen$\mathrm{de}^{72}$ waren unzweifelhaft auch intellektuelle Schockreaktionen und Ausdruck des verzweifelten Versuchs, der in ihrer zunehmenden Dynamik dann doch so überraschenden politischen Wende wenigstens mit Stellungnahmen und Neupositionierungen zu antworten. Fragen nach Vergangenheitsbewältigung und Schuld, nach Gemeinsamem und Fremdem, nach Zivilisation und Kultur erhielten eine neue Bedeutung und bestimmen den zeitgenössischen Diskurs nachhaltig. Die Kulturwissenschaft gehörte zu einer der wenigen Errungenschaften der DDR, die nach der Wende und den Abwicklungen innerhalb der Universitäten unter Beibehaltung der Denomination bei gleichzeitiger inhaltlicher Umgestaltung und Expansion des Faches übernommen worden ist. ${ }^{73}$

Die interkulturelle Literaturwissenschaft sah sich durch die Wiedervereinigung vor die Aufgabe gestellt, die Unterscheidung von Fremdem

\footnotetext{
72 In der Literaturdebatte ging es einerseits um Christa Wolf, deren Mitarbeit bei der Stasi bekannt wurde. Vgl. Hermann Vinke: Akteneinsicht Christa Wolf. Zerrspiegel und Dialog. Eine Dokumentation. Hamburg: Luchterhand 1993. Mit den Rezensionen zu ihrer Erzählung Was bleibt entbrannte dann eine heftige Debatte. Thomas Anz (Hg.): Es geht nicht um Christa Wolf. Der Literaturstreit im vereinten Deutschland. Frankfurt a.M.: Fischer 1995. Andererseits wurde die Literaturdebatte dann fortgesetzt mit: Botho Strauss: "Anschwellender Bocksgesang." In: Heimo Schwilk, Ulrich Schacht (Hg.): Die selbstbewußte Nation. "Anschwellender Bocksgesang" und weitere Beiträge zu einer deutschen Debatte. Frankfurt a.M.: Ullstein 1996,19-40.

${ }^{73}$ Vgl. Hartmut Böhme, Peter Matussek, Lothar Müller: Orientierung Kulturwissenschaft. Was sie kann, was sie will. Reinbek bei Hamburg: Rowohlt 2000.
} 
und Eigenem unter intrakultureller Perspektive neu zu durchdenken. ${ }^{74}$ Angezeigt war damit also auch ein Perspektivenwechsel, denn es ging nun nicht mehr um die Auseinandersetzung mit der deutschen Literatur unter fremdsprachlicher Perspektive, sondern implizit um ein FremdWerden oder Fremd-werden-Lassen des eigenen Selbstverständnisses. Die so gewonnene Distanz vom scheinbar Vertrauten ist Voraussetzung einer umfassenden Selbstreflexion und Neuorientierung des Faches, mit der nun verstärkt Konstruktionsprinzipien von Differenz ins Blickfeld rücken.

Innerhalb kulturwissenschaftlicher Forschung wird Kultur je nach Untersuchungsansatz als Netz von Bedeutungen gefasst, in das der Mensch selbst verstrickt ist, ${ }^{75}$ oder als "Komplex von Werten, Sitten und Gebräuchen, Überzeugungen und Praktiken, die die Lebensweise einer bestimmten Gruppe ausmachen"76 oder aber als "eine Konstellation von Texten, die - über das geschriebene oder gesprochene Wort hinaus - auch in Ritualen, Theater, Gebärden, Festen usw. verkörpert sind." Bestimmungen von Kultur ist gemeinsam, dass nach Symbolisierungsformen gesucht wird, durch die sich eine soziale Gemeinschaft in ihrer kulturellen Identität selbst beglaubigt. Interkulturelle Literaturwissenschaft denkt Kultur demgegenüber nicht als fest umgrenzte Entität, sondern geht von den Interaktionsprozessen aus, bei denen die kulturelle Differenz zwischen eben diesen Werten, Sitten, Gebräuchen und Praktiken als kulturkonstitutiv verhandelt werden.

Untersucht wird "Kultur als Text" im Sinne einer Vertextung wechselseitiger Bedeutungszuschreibung, als Intertextualität zwischen Kulturen.

\footnotetext{
${ }^{74}$ Vgl. die Beiträge von Lutz Henner Richter, Walter Hinderer, Ulrich Merkel, Hubert Orlowski und Amadou Booker Sadji aus der Sektion "Die Erfahrung der 'Wende' Konsequenzen für die interkulturelle Arbeit." In: Bernd Thum, Gonthier-Louis Fink (Hg.): Praxis interkultureller Germanistik. Forschung-Bildung-Politik. Beiträge zum II. Internationalen Kongreß der Gesellschaft für Interkulturelle Germanistik; Strasbourg 1991. München: Iudicium 1993, 195-240.

${ }^{75}$ Clifford Geertz: Dichte Beschreibung. Beiträge zum Verstehen kultureller Systeme. Frankfurt a.M.: Suhrkamp 1983, 9ff.

${ }^{76}$ Terry Eagleton: Was ist Kultur?: eine Einführung. München: Beck 2001, 51.

${ }^{77}$ Doris Bachmann-Medick: "Einleitung." In: Dies. (Hg.): Kultur als Text. Die anthropologische Wende in der Literaturwissenschaft. Frankfurt a.M.: Fischer 1996, 7-64, hier: 10.
} 
Interkulturalität meint also nicht Interaktion zwischen Kulturen im Sinne eines Austausches von je kulturell Eigenem, sondern zielt auf ein intermediäres Feld, das sich im Austausch der Kulturen als Gebiet eines neuen Wissens herausbildet. So wird mit Begriff Interkulturalität eine Grenzüberschreitung in den Blick genommen, bei der weder ein wie auch immer gefasstes Innerhalb oder Außerhalb der Grenze, noch die Grenze zum eigentlichen Untersuchungsgegenstand wird, sondern vielmehr das Inter selbst. Mithin geht es also um die Untersuchung der Funktionsweise von Differenzbestimmungen, die Kulturationsprozesse abstützen, verändern oder neu in Gang setzen.

Mit der interkulturellen Literaturwissenschaft kommt also ein prozesshafter und dialogischer Kulturbegriff zur Anwendung, der an der Selbstthematisierungsfähigkeit von Gesellschaft ansetzt und die kontextbezogene Veränderung von Bedeutungen und Handlungen zwischen Menschen, die sich situativ und multipel verorten, untersucht. In diesem Oszillieren zwischen unterschiedlichen Handlungsorientierungen kommt es zur Auflösung und Neuschaffung von Grenzziehungen, Macht- und Gewaltverhältnissen und Geschlechterrollen. Die Untersuchung interkultureller Kommunikation bezieht sich somit auf kommunikative Akte zwischen Personen, die sich mittels kultureller Zeichen als voneinander unterschiedlich identifizieren. ${ }^{78}$ Interkulturelle Literaturwissenschaft reflektiert auf eben diese Verfahren der Bedeutungszuschreibung. Damit rückt aber auch die kulturspezifische Konstruktion von Geschlecht und die Verfahren der geschlechtsspezifischen Bedeutungszuschreibung ins Zentrum der interkulturellen Literaturwissenschaft.

So fanden beispielsweise im Rahmen des durch die Deutsche Forschungsgemeinschaft geförderten Projektes "Das Subjekt und die Anderen. Interkulturalität und Geschlechterdifferenz von der frühen Neuzeit bis zur Gegenwart" an der Universität Trier Tagungen statt, in denen die europäische Subjektkonstruktion über die Etablierung jeweiliger "Ande-

\footnotetext{
${ }^{78}$ Vgl. Gabriel Layes: Grundformen des Fremderlebens. Eine Analyse von Handlungsorientierungen in der interkulturellen Interaktion. Münster u. a.: Waxmann 2000.
} 
rer" untersucht wurde. ${ }^{79}$ Hier wurde der Versuch unternommen, die Interkulturalitätsforschung und die feministische Kulturkritik zusammenzuführen und Überschneidungen von kultureller und sexueller Differenz in den Blick zu nehmen. Das Forschungsinteresse richtete sich auf Erzeugungsregeln sexueller, ethnischer und nationaler Differenz in literarischen Repräsentationen. Dabei ging es auch um die Frage, in welcher Weise Geschlechtermythen kulturelle Unterschiede und Machtverhältnisse naturalisieren. Untersucht wurde, wie Deutungsmuster des sexuell und kulturell Fremden durch Ethnisierung hierarchisiert werden. Insofern Kulturbegegnung nicht selten in den Dichotomien von männlich und weiblich gefasst wird, wurde innerhalb dieses Forschungszusammenhanges herausgearbeitet, dass die Selbstermächtigung der Europäer über Geschlechtsmetaphern konstruiert wird. ${ }^{80}$ Die Begegnung zwischen alter und neuer Welt ist nicht selten in das Narrativ einer Paarbeziehung eingekleidet, mit dem koloniale Beziehungen als natürlich gegebene verbrämt werden. ${ }^{81}$ Innerhalb der Literaturwissenschaft wurde in diesem Zusammenhang Heinrich von Kleists Die Verlobung in St. Domingo zu einem Schlüsseltext, an dem die ästhetische Verfasstheit des Ineinandergreifens von kultureller und sexueller Differenz vielfach untersucht wurde. ${ }^{82}$ Ansatzpunkt ist, dass in der Literatur jene Dichotomien, welche Kultur strukturieren, mit ihren Verwerfungen und Verfehlungen, und damit auch in Distanz zum Diskurs, inszeniert werden. Während in der feministischen Literaturwissenschaft die Frau in ihrem Objektstatus untersucht

\footnotetext{
${ }^{79}$ Z. B. Herbert Uerlings, Karl Hölz, Viktoria Schmidt-Linsenhoff (Hg.): Das Subjekt und die Anderen: Interkulturalität und Geschlechterdifferenz vom 18. Jahrhundert bis zur Gegenwart. Berlin: Erich Schmidt 2001.

${ }^{80} \mathrm{Vgl}$. hierzu auch: Tzvetan Todorov: Die Eroberung Amerikas. Das Problem der Anderen. Frankfurt a.M.: Suhrkamp 1985, 185.

${ }^{81}$ Vgl. hierzu ausfuhrlich: Herbert Uerlings: "Das Subjekt und die Anderen. Zur Analyse sexueller und kultureller Differenz. Skizze eines Forschungsbereichs." In: Ders., Hölz, Schmidt-Linsenhoff: Das Subjekt und die Anderen, 19-54, hier: 19.

${ }^{82}$ Als Beispiele: Anselm Haverkamp: "Schwarz, Weiß: 'Othello' und 'Die Verlobung in St. Domingo." In: Weimarer Beiträge 41.3 (1995), 397-409. Hansjörg Bay: "'Als die Schwarzen die Weißen ermordeten': Nachbeben einer Erschütterung des europäischen Diskurses in Kleists 'Verlobung in St. Domingo."' In: Kleist-Jahrbuch 1998, 80-108. Paul Michael Lützeler: Napoleons Kolonialtraum und Kleists 'Die Verlobung in St. Domingo.' Wiesbaden: Westdt. Verl. 2000. Ders.: "Verfuhhrung und Missionierung: zu den Exempeln in 'Die Verlobung in St. Domingo.'” In: Ders., David Pan (Hg.): Kleists Erzählungen und Dramen. Würzburg: Königshausen \& Neumann 2001, 35-48.
} 
wurde, gerät bei der Forschergruppe an der Universität Trier das hegemoniale Subjekt in den Blick, das des konstitutiven Fremden bedarf, um sich als solches überhaupt etablieren zu können. ${ }^{83}$ Im Folgenden werde ich jedoch die Verbindung von Gender und Interkulturalität unter der Perspektive der Differenzkonstruktion darlegen.

Zunächst einmal fungiert der Begriff Interkulturalität im allgemeinen Sprachgebrauch als Beschreibungskategorie, mit welcher das Aufeinandertreffen unterschiedlicher kultureller Horizonte und Ansprüche bezeichnet wird. Mithin werden damit Reaktionsweisen auf kulturelle Alterität gefasst. Interkulturalitätsforschung setzt bei diesen Reaktionsformen als Konstruktionsprinzipien an. Denn worauf in der interkulturellen Kommunikation vermeintlich nur reagiert wird, wird in seiner Bedeutung durch die Interaktion erst hergestellt. Das Forschungsparadigma Interkulturalität rückt damit die Unterschiedlichkeit von Kultur als interpretative Leistung sozialer Subjekte ins Zentrum. Diese interpretative Leistung aber basiert auf einer Relation: auf der Konstruktion eines Zuschreibungsverhältnisses von Eigenem und Fremdem.

Fremde ist nun aber keine vorfindbare Gegebenheit oder Eigenschaft, die einem Objekt zukommt und bezeichnet auch keine objektiv messbare Größe. Das Fremde ist vielmehr ein Relations- oder Unterscheidungsbegriff zum Eigenen und somit ohne das Eigene gar nicht denkbar, und umgekehrt, wie dies in der Fremdheitsforschung verschiedentlich dargelegt wurde. Häufig ist den Beteiligten gar nicht bewusst, dass es sich bei der Bestimmung von Fremdem und Eigenem um Definitionen handelt, da ihnen die Unterscheidung als naturwüchsige Differenzen erscheinen. Dabei sind es nicht die Unterschiede, die jemanden zum Fremden machen, sondern es ist eine institutionalisierte Fremde, die zur Wahrnehmung und Dramatisierung von Unterschieden führt. Das Eigene und das Fremde sind heuristisch als operationale Größen von Interkulturalität $\mathrm{zu}$ verstehen, die also erst durch die Operationalisierung

\footnotetext{
${ }^{83}$ Vgl. hierzu auch: Uerlings: Das Subjekt und die Anderen, 23. Für die deutschsprachige Forschung siehe z. B. Sedef Gümen: "Die sozialpolitische Konstruktion 'kultureller' Differenzen in der bundesdeutschen Frauen- und Migrationsforschung." In: Beiträge zur feministischen Theorie und Praxis 42 (1996), 77-90.
} 
hervorgebracht werden. Die Differenz zwischen Eigenem und Fremdem lässt sich als Bedeutungszuschreibung verstehen, die an den jeweiligen kulturellen Kontext gebunden ist und innerhalb dieses Kontextes plausibel und anschließbar sein muss.

Das Fremde ist demnach Teil einer kulturdistinktiven Beziehungsdefinition, ${ }^{84}$ die erst Selbstdefinition ermöglicht, da jede Selbstbeschreibung Alterität, von der sich das Selbst abgrenzend profiliert, in Anspruch nehmen muss. Als Begriff, der in der Philosophie, Ethnologie, Soziologie, Psychologie und Anthropologie mit unterschiedlicher Akzentuierung zur Erklärung von Selbstkonstitution in Interaktionsprozessen herangezogen wird, umfasst Alterität die Vorstellung von einem in Bezug auf das Eigene gleichursprünglich Anderen. Insbesondere der philosophische Ansatz von Emmanuel Lévinas wurde für die begriffliche Bestimmung von Alterität interdisziplinär fruchtbar. Nach Lévinas' Bestimmung ist der Andere "nicht zunächst Gegenstand des Verstehens und dann Gesprächspartner," sondern "beide Beziehungen" fallen zusammen: "Vom Verstehen des Anderen ist seine Anrufung untrennbar." ${ }^{, 85}$ In dieser unhintergehbaren Gebundenheit an den Anderen ist Identitätsfindung auf die immer neue Inszenierung des Beziehungsverhältnisses verwiesen.

Diese Form der Inszenierung kann als anthropologisches Grundmuster für die Herstellung von Selbstidentität verstanden werden. Der intermediäre Raum zwischen dem Anwesenden und dem Abwesenden kann als "potential space," 86 als Ort des kulturellen Erlebens, ausgemacht werden. Denn mit dem Gewahrwerden des Getrenntseins von anderen Objekten mit der gleichzeitigen Etablierung von Anderem und Ich, geht auch

\footnotetext{
${ }^{84}$ Alois Hahn: "Die soziale Konstruktion des Fremden." In: Walter M. Sprondel (Hg.): Die Objektivität der Ordnungen und ihre kommunikative Konstruktion. Frankfurt a.M.: Suhrkamp 1994, 140-166, hier: 140. Kai Uwe Hellmann: "Fremdheit als soziale Konstruktion. Eine Studie zur Systemtheorie des Fremden." In: Herfried Münkler (Hg.): Die Herausforderung durch das Fremde. Berlin: Akademie 1998, 401-459.

${ }^{85}$ Emmanuel Lévinas: "Ist die Ontologie fundamental?" In: Ders.: Die Spur des Anderen. Untersuchungen zur Phänomenologie und Sozialphilosophie. Freiburg, München: Alber 1983, 103-119, hier: 111.

${ }^{86}$ Donald W. Winnicott: "Zur Lokalisierung des kulturellen Erlebens." In: Ders.: Vom Spiel zur Kreativität. Stuttgart: Klett-Cotta 1985,111-120; hier: 116.
} 
immer der Entwurf eines kreativen Spannungsfeldes einher. In diesem Möglichkeitsraum wird die Grenze zwischen Eigenem und Anderem spielerisch exploriert und das Probehandeln gewinnt schöpferischen Gestaltungscharakter für die Differenzbestimmung zwischen dem Selbst und dem Anderen. Unter dem Aspekt der Interkulturalität inszeniert Literatur dieses anthropologische Grundmuster im Pendeln zwischen eigen- und fremdkulturellem Bezugsfeld immer wieder neu und eröffnet somit den für die Welt- und Selbsterfahrung konstitutiven Möglichkeitsraum.

Ich möchte dafür plädieren, auch Gender in diesem strukturellen Sinne zu verstehen. Denn auch Männlichkeit und Weiblichkeit sind Relationsoder Unterscheidungsbegriffe, mit denen Differenzpositionen bestimmt werden. In der Rede von Weiblichkeit wird Männlichkeit als das gleichursprünglich andere gesetzt und umgekehrt. Weiblichkeit und Männlichkeit sind demnach heuristisch als operationale Größen von Geschlechtlichkeit zu verstehen, die erst durch die kulturellen Prozesse der Vergeschlechtlichung hervorgebracht werden. Auch die geschlechtliche Differenzierung ist daher an den jeweiligen kulturellen Kontext gebunden und nur innerhalb dieses Kontextes plausibel.

Auf Geschlecht wird somit nicht reagiert, sondern es wird in seiner Bedeutung durch kommunikative Situationen hergestellt. Geschlecht basiert auf keiner naturwüchsigen Differenz, sondern die Differenz wird als Bedeutungsraum, in dem Geschlechtlichkeit zugesprochen wird, erst hergestellt. Somit sind es nicht vorfindbare Unterschiede, die durch die Begriffe Männlichkeit und Weiblichkeit nur ihren sprachlichen Ausdruck finden, sondern es ist eine Tradition von geschlechtsspezifischen $\mathrm{Zu}$ schreibungen, durch die Geschlechterdifferenzen wahrgenommen und dramatisiert werden. Geschlechtlichkeit wird einerseits durch einen kulturellen Kanon von Zuschreibungen bestimmt und andererseits gleichzeitig durch performative Akte, mit denen Teile des kulturellen Archivs fortgeschrieben, umgedeutet oder subvertiert werden. Geschlechtlichkeit ist somit kulturelles Archiv, das über Speichermedien wie Bilder, Texte, Tonaufzeichnungen und Filme präsent gehalten wird. Zugleich aber ist dieses Archiv auch Fundus, mit dessen Hilfe Geschlecht immer wieder neu inszenierbar wird. Dramatisiert aber werden Geschlechtsunterschiede 
besonders in Phasen kulturellen Wandels, insofern Geschlechterzuschreibungen ins Wanken geraten und umcodiert werden. Das Geschlecht ist demnach wie das Fremde Teil einer kulturdistinktiven Beziehungsdefinition, ${ }^{87}$ die erst Selbstdefinition ermöglicht, da jede Selbstbeschreibung geschlechtsspezifische Alterität, von der sich das Selbst abgrenzend profiliert, in Anspruch nehmen muss.

Für die Gender-Studien ergeben sich im Zusammenhang mit der interkulturellen Literaturwissenschaft neue Forschungsfelder. Auch wenn die interkulturelle Literaturwissenschaft verglichen mit anderen Fächern und Teildisziplinen erst auf eine kurze Wissenschaftsgeschichte zurückblicken kann, so zeichnen sich doch bereits Entwicklungstendenzen ab. Kennzeichnend für die Anfangsphase sind zahlreiche Publikationen, die sich der Selbstdefinition und Profilierung des Faches gegenüber den Nachbardisziplinen widmen oder aber überhaupt den fächerübergreifenden Ansatz von Interkulturalität als neues Forschungsparadigma betonen. Mit der interkulturellen Hermeneutik, der Kulturthemenforschung, ${ }^{88}$ der Imagologie und der Stereotypenforschung hat sich die interkulturelle Literaturwissenschaft darüber hinaus ein breit gefächertes Forschungsfeld erschlossen, bei dem die Untersuchung von kulturspezifischen Zuschreibungsverhältnissen zentral sind. In ihren theoretischen Ansätzen orientiert sich die interkulturelle Literaturwissenschaft deshalb auch an jenen Disziplinen und Forschungsrichtungen, ${ }^{89}$ die sich der Untersuchung von Formen und Prinzipien der Kulturentwicklung und Differenzkonstruktionen widmen wie die Ethnographie, ${ }^{90}$ die Kultur- und Sozialanthropo-

\footnotetext{
${ }^{87}$ Vgl. Hahn, Die soziale Konstruktion des Fremden, 140-166. Kai Uwe Hellmann: "Fremdheit als soziale Konstruktion. Eine Studie zur Systemtheorie des Fremden." In: Herfried Münkler (Hg.): Die Herausforderung durch das Fremde. Berlin: Akademie 1998, 401-459.

${ }^{88}$ Vgl. Bernd Thum: "Einleitung." In: Ders. (Hg.): Gegenwart als kulturelles Erbe. Ein Beitrag der Germanistik zur Kulturwissenschaft deutschsprachiger Länder. München: Iudicium 1985, XV-LXVII, hier; insbesondere Kap. 3: "Aufgaben im Rahmen allgemeiner Kulturwissenschaft: 'Kulturthemen," XXXII ff.

${ }^{89}$ Ortrud Gutjahr: "Interkulturalität und Interdisziplinarität." In: Christine Maillard, Arlette Bothorel-Witz (Hg.): Du dialogue des disciplines: germanistique et interdisciplinarité. Strasbourg: Presses Univ. de Strasbourg 1998, 135-149.

${ }^{90}$ Besonders einflussreich waren hier die Untersuchungen und Bestimmungen von Clifford Geertz sowie Stephen Greenblatt: Schmutzige Riten. Berlin: Wagenbach 1991. Dazu: Lutz
} 
logie, die kulturtheoretisch orientierte Ethnopsychoanalyse ${ }^{91}$ und Psychohistorie, ${ }^{92}$ die interkulturell orientierte Gender-Forschung ${ }^{93}$ und die genuin interkulturell orientierte Fremdheitsforschung.

Bei der Frage nach kulturspezifischen Bedeutungszuschreibungen ist die interkulturelle Literaturwissenschaft prinzipiell offen für alle literarische Gattungen. Ins Zentrum des Interesses rücken aber besonders kulturreflexive Gattungen wie Reise-, Kolonial-, Exil- und Migrationsliteratur, Utopien, Abenteuerromane und Robinsonaden sowie die postkoloniale Literatur, in der die kolonialen Begegnungen und ihre Folgen oftmals von Autorinnen und Autoren aus früheren Kolonialgebieten in der Sprache der einstmaligen Kolonialmächte thematisiert sind. ${ }^{94}$ Materiale Untersuchungsbasis der interkulturellen Literaturwissenschaft sind also Texte und Textgattungen, in denen die ästhetische Inszenierung und Reflexion der unterschiedlichen Formen und Konflikte der Kulturbegegnung konstitutiv șind. ${ }^{95}$ Die Auseinandersetzung mit interkulturellen Fragestellungen schuldet dabei ihre Brisanz einer methodischen Notwendigkeit. Die Kontextualisierung der literarischen Texte in ihrem jeweiligen epochen- und kulturspezifischen Diskursfeld wird zugleich zu einer spezifischen Form der Arbeit am kulturellen Gedächtnis. Denn die europäische Kultur ist, wie dies durch den Begriff Cultural Negotiation gefasst wird, durch spezifische Formen der Interkulturalität gekennzeichnet, durch "Wechselwirkungen und Strategien der Beeinflussung" und die Absorbierung fremder

\footnotetext{
Ellrich: Verschriebene Fremdheit. Die Ethnographie kultureller Brüche bei Clifford Geertz und Stephen Greenblatt. Frankfurt a.M., New York: Campus 1998.

${ }^{91}$ Mario Erdheim: Die gesellschaftliche Produktion von Unbewußtheit. Eine Einführung in den ethnopsychoanalytischen Prozeß. Frankfurt a.M.: Suhrkamp 1984 sowie Evelyn Heinemann, Günter Krauss (Hg.): Beiträge zur Ethnopsychoanalyse. Der Spiegel des Fremden. Nürnberg: Inst. für Soziale und Kulturelle Arbeit 1992.

${ }^{92}$ Ulrich Streek (Hg.): Das Fremde in der Psychoanalyse. Erkundungen über das 'Andere' in Seele, Körper und Kultur. München: Pfeiffer 1993.

${ }^{93} \mathrm{Hölz}$, Schmidt-Linsenhoff, Uerlings, Das Subjekt und die Anderen

${ }^{94}$ Ferdinand Dennis, Naseem Khan (Hg.): Voices of the Crossing. The Impact of Britain on Writers from Asia, the Caribbean and Africa. London: Serpent's Tail 2000.

${ }^{95}$ James Axtell: Natives and Newcomers. The Cultural Origins of North America. New York, Oxford: Oxford UP 2001. Anil Bhatti, Horst Turk (Hg.): Reisen, Entdecken, Utopien. Untersuchungen zum Alteritätsdiskurs im Kontext von Kolonialismus und Kulturkritik. Bern u. a.: Lang 1998.
} 
Kultur bei der "Strukturierung der eigenen." Kultur ist nicht nur durch die großen inner- und interkontinentalen Wanderbewegungen geprägt, sondern auch durch die europäischen Formen der Welterkundung, die in weiten Teilen mit räumlicher Expansion, Ausbeutung, Zerstörung und der Unterordnung anderer Erfahrungswelten und Traditionen unter die Perspektive eigener Weltsicht einhergehen ${ }^{97}$ und deshalb mit der Schuldfrage verknüpft sind. ${ }^{98}$ Die Kartographierung der Erde durch die Europäer geht mit Benennung, Klassifizierung, Bewertung und Ermächtigung einher, die sich bis in den heutigen Sprachgebrauch von erster, zweiter und dritter Welt wiederspiegelt.

Theorien zum Eurozentrismus und Rassismus oder Kolonialismus, die einem interkulturellen Ansatz verpflichtet sind, lassen sich vor diesem Hintergrund auch als Beitrag zur Kritik der Moderne verstehen. ${ }^{99}$ Einen genuin interkulturellen Ansatz vertreten diesbezüglich auch postkoloniale Theorien, ${ }^{100}$ bei denen es um die Strukturbedingungen der imperialen Vereinnahmungen geht, wie bei Edward Said, ${ }^{101}$ oder um die Anerkennung $^{102}$ des postkolonialen Subjekts in einer zwischen den Kulturen angesiedelten Situation, wie bei Homi Bhabha. Mit der Denkfigur des third space hat Bhabha dem intermediären Feld zwischen den Kulturen als dem Raum der kulturellen Verortung des postkolonialen Subjekts eine neue Bestimmung eingeschrieben. Eingefordert wird die Anerkennung einer hybriden Existenzform, bei der sich Subjekte kulturell multipel und situativ selbst bestimmen können. Damit wird auf einer kulturellen Dif-

\footnotetext{
${ }^{96}$ Therese Fischer-Seidel: "Vorwort." In: Cedric Brown, Dies. (Hg.): Cultural Negotiations Sichtweisen des Anderen. Tübingen u. a.: Francke 1998, 7f, hier: 8.

${ }^{97}$ Vgl. Jared Diamond: Arm und Reich. Die Schicksale menschlicher Gesellschaften. Frankfurt a.M.: Fischer 1997.

${ }^{98}$ Vgl. Urs Bitterli: Die 'Wilden' und die 'Zivilisierten.' Grundzüge einer Geistes- und Kulturgeschichte der europäisch-überseeischen Begegnung. München: Beck 1976.

${ }^{99}$ Vgl. Thomas K. Nakayama, Judith N. Martin (Hg.): Whiteness. The Communication of Social Identity. Thousand Oaks: Sage Publications 1999.

${ }^{100}$ Vgl. Bill Ashcroft, Gareth Griffiths, Helen Tiffin (Hg.): The Post-Colonial Studies Reader. London, New York: Routledge 1995.

${ }^{101} \mathrm{Vgl}$. Edward W. Said: Kultur und Imperialismus. Einbildungskraft und Politik im Zeitalter der Macht. Frankfurt a.M.: Fischer 1994 [Culture and Imperialism. New York: Knopf 1993].

102 Vgl. Axel Honneth: Kampf um Anerkennung. Zur moralischen Grammatik sozialer Konflikte. Frankfurt a.M.: Suhrkamp 1992.
} 
ferenz insistiert, die gerade nicht über die Relation zu einer wie auch immer bestimmten Alterität festgeschrieben, sondern zur produktiven Verunsicherung offen gehalten werden soll. ${ }^{103}$ Gerade solche Ansätze lassen sich auch unter Gender-Perspektive operationalisieren. Denn der dritte Raum verweist nicht nur auf das intermediäre Feld von Interkulturalität und die prinzipielle Unabgeschlossenheit kultureller Systeme, sondern auch der Geschlechterzuschreibung. ${ }^{104}$

So werden die Analysen zur kulturellen Konstruktion von Geschlecht für eine interkulturelle Gender-Theorie anschlussfähig, wenn die Kategorie Geschlecht auch als interkulturell, translokal und multipel konstruiert untersucht wird. ${ }^{105}$ Auch Judith Butler hat in ihren Untersuchungen herausgestellt, dass kulturelle Geschlechtskonstruktionen über Ausschluss-bestimmungen vermittelt sind: "und zwar so, daß das Menschliche nicht nur in Abgrenzung gegenüber dem Unmenschlichen produziert wird, sondern durch eine Reihe von Verwerfungen, radikalen Auslöschungen, denen die Möglichkeit kultureller Artikulation regelrecht verwehrt wird." 106 Interkulturelle Literaturwissenschaft richtet ihr Augenmerk dabei auf die Verwerfungen ${ }^{107}$ bei interkulturellen Geschlechterbeziehungen, die von der antiken Mythologie bis zur Gegenwartsliteratur im Zusammenhang mit Kriegen, Handel, Kolonialismus und Reisen nicht selten im Zeichen von Gewalt und rigiden Grenzziehungen stehen. Auch die Erzeugungsregeln solcher Grenzziehungen und damit die Ein- und Ausschlussbewegungen zu untersuchen, nach denen sich Kultur formiert, ist

\footnotetext{
${ }^{103}$ Vgl. Homi K. Bhabha: Die Verortung der Kultur. Tübingen: Stauffenburg 2000 [The Location of Culture. London, New York: Routledge 1994].

104 Doris Bachmann-Medick: "Dritter Raum. Annäherungen an ein Medium kultureller Übersetzung und Kartierung." In: Claudia Breger, Tobias Döring (Hg.): Figuren der/des Dritten. Erkundungen kultureller Zwischenräume. Amsterdam, Atlanta: Rodopi 1998, 1936.

105 Judith Schlehe: "Gender als transkulturelle Konstruktion." In: Judith Schlehe (Hg.): Zwischen den Kulturen - Zwischen den Geschlechtern. Kulturkontakte und Genderkonstrukte. Münster u. a.: Waxmann 2000, 7-16.

106 Judith Butler: Körper von Gewicht. Die diskursiven Grenzen des Geschlechts. Berlin: Berlin-Verl. 1995, 29.

${ }^{107} \mathrm{Vgl}$. James P. Sterba: Three Challenges to Ethics. Environmentalism, Feminism and Multiculturalism. New York u. a.: Oxford UP 2001.
} 
Aufgabe einer Gender-Forschung innerhalb der interkulturellen Literaturwissenschaft. 